\title{
Need to bankroll efforts to roll back malaria
}

Our understanding of the biology of Plasmodium falciparum and its interaction with the human host will be refreshed by the recent description of the parasite's complete genome sequence, leading to new approaches and targets for the discovery of drugs and vaccines, according to a report in Nature. However, the impact of this information on the malaria epidemic may be restricted by the rate-limiting step of acquiring funds and industrial expertise to advance promising new drugs and vaccines into the marketplace.

This lack of finance and an excess of information from the $P$. falciparum genome is likely to result in increased tension between those who support investing in the development of new drugs and vaccines, and those who want the money to go towards improving existing methods of control, suggests the report. The timescale required to generate practical agents for malaria control from genomics information must also be considered. Against expectations that the $P$. falciparum genome should rapidly generate new targets that can quickly proceed into clinical trials, some malaria researchers believe it could take up to two decades to convert genomic data into effective vaccines, the report says. According to Tikki Pang, the WHO's director of research policy and cooperation, rigorous, independent, costbenefit analyses are required to shed light on the debate, says the report.

The report concludes that 'while biomedical scientists and public-health experts argue over the relative merits of investing in genomics, drug and vaccine development, or the application of existing control methods, what's really needed is more money across the board . . . The stark truth is: if we don't bankroll the effort, we won't roll back malaria'.

What difference does a genome make? Nature 419: 426-428, 3 Oct 2002 